Einführung zum Thema

Orthopäde 2020 · 49:941

https://doi.org/10.1007/s00132-020-03989-3

(c) Springer Medizin Verlag $\mathrm{GmbH}$, ein Teil von Springer Nature 2020

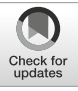

Jörn Dohle

OGAM Orthopädie, Wuppertal, Deutschland

\title{
Der erworbene Plattfuß des Erwachsenen
}

sidueller Planovalgusdeformitäten des jungen Erwachsenen.

Aber auch bei der Behandlung früherer Stadien der Pathologie ist zu hinterfragen, ob der Tibialis-posterior-Sehne nicht doch eine Regenerationsfähigkeit unterstellt werden darf. Das Konzept des Debridements der Tibialis-posterior-Sehne durch Tenoskopie in Kombination mit einer mechanischen Entlastung durch medialisierende Kalkaneusverschiebeosteotomie und Arthrorise des Subtalargelenks unterstellt bzw. basiert auf genau dieser Regenerationsfähigkeit der Sehne.

Vor einer operativen Korrektur muss als erstes die Frage beantwortet werden, ob noch eine flexible oder schon eine kontrakte Deformität vorliegt. Für die flexiblen Deformitäten ist die Kombination aus motorischer Ersatzplastik, z. B. durch Transposition der Flexor-digitorum-longus-Sehne auf den medialen Navikularepol, und Korrektur des Fersenvalgus durch eine medialisierende Verschiebeosteotomie des Tuber calcanei der de facto Standard. Rigide Deformitäten werden dagegen durch eine Korrekturarthrodese des Rückfußes stabilisiert.

Der ehemals goldene Standard der Triple-Arthrodese wird heute zugunsten der Double-Arthrodese, also der kombinierten Arthrodese von Talonavikulargelenk und Subtalargelenk verlassen. Strittig ist weiterhin, wie häufig nach einer Double- oder Triple-Arthrodese eine sekundäre Arthrose des Sprunggelenks erwartet werden muss. Mittelfristige Verlaufsbeobachtungen lassen zu mindestens eine radiologische Progression degenerativer Veränderungen des oberen Sprunggelenks erkennen.

Durch die Kombination multipler Osteotomien des Rückfußes und einer Kor- rektur des Torsionsprofils der Fußsohle durch Mittelfußosteotomie und/oder Mittelfußarthrodesen, scheint es möglich, das Indikationsspektrum operativer Korrekturen unter Erhalt einer ,gewissen Restbeweglichkeit" des unteren Sprunggelenks auszuweiten und somit das Risiko einer sekundären Arthrose des oberen Sprunggelenks weiter zu reduzieren.

Selbst in Fälle, in denen bereits sekundäre Veränderungen des oberen Sprunggelenks eingetreten sind, sei es in Form von degenerativer Veränderung oder in Form ligamentärer Instabilitäten, ist ein Erhalt des oberen Sprunggelenks häufig noch möglich. Eine vollständige Korrektur der subtalaren Fehlstellung ist dann unabdingbar und muss mit Verfahren der Knorpelrekonstruktion und mit Bandrekonstruktionen kombiniert werden. Dabei handelt es sich zweifelsohne um hochkomplexe Eingriffe, die vor allem bei jüngeren Patienten in spezialisierten Zentren durchgeführt werden sollten.

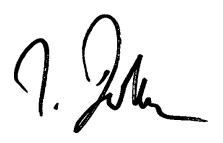

Dr. J. Dohle

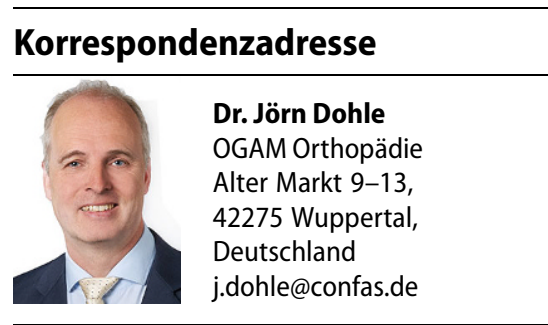

Interessenkonflikt. J. Dohle gibt an, dass kein Interessenkonflikt besteht. 